\title{
Development of a multiple-choice problem-solving categorization test for assessment of student knowledge structure
}

\author{
Qingwei Chen $\odot,{ }^{1,2}$ Guangtian Zhu, ${ }^{1, \dagger}$ Qiaoyi Liu, ${ }^{2}$ Jing Han, ${ }^{2}$ Zhao Fu, ${ }^{2}$ and Lei Bao $\odot^{2, *}$ \\ ${ }^{1}$ College of Teacher Education, East China Normal University, Shanghai 200062, China \\ ${ }^{2}$ Department of Physics, The Ohio State University, Columbus, Ohio 43210, USA
}

(Received 6 June 2020; accepted 25 August 2020; published 11 September 2020)

\begin{abstract}
Problem-solving categorization tasks have been well studied and used as an effective tool for assessment of student knowledge structure. In this study, a traditional free-response categorization test has been modified into a multiple-choice format, and the effectiveness of this new assessment is evaluated. Through randomized testing with Chinese college students, the multiple-choice categorization test has been shown to provide equivalent measurement compared to the open-ended test. In addition, the influence of including diagrams in problems on students' categorization performances has also been studied, which suggests that the inclusion of diagrams in problems can improve students' performances in identifying the relevant concepts. The results also show that the knowledge structures of the Chinese college students are in the early transitional stage between novices and experts.
\end{abstract}

DOI: 10.1103/PhysRevPhysEducRes.16.020120

\section{INTRODUCTION}

A main goal of science, technology, engineering, and math (STEM) education nowadays is to prepare students to solve real-world problems like experts, which requires them to develop and apply deep understandings of science principles and concepts. However, students going through traditional instruction are often found to stay at the novice level, in which learners primarily rely on memorizing rules and algorithms in problem solving and lack the desired deep conceptual understanding, even though they may be well versed in solving traditional problems [1-3].

As a result, in teaching and learning it is crucial to distinguish experts and novices and identify the means to transform novices to experts. Two key aspects that differentiate experts and novices are the connectedness and context dependence of their knowledge structures. Experts have an integrated knowledge structure, where ideas are hierarchically organized as a network around a few key principles, with many consistent and long-range links around these ideas from concrete surface features deep into the abstract domain [4-9]. Therefore, experts are able to see past the surface features during problem solving,

\footnotetext{
Corresponding author. bao.15@osu.edu

Corresponding author. gtzhu@phy.ecnu.edu.cn

Published by the American Physical Society under the terms of the Creative Commons Attribution 4.0 International license. Further distribution of this work must maintain attribution to the author(s) and the published article's title, journal citation, and DOI.
}

and recognize the key variables across different domains and in unfamiliar contexts $[4,10]$. Conversely, novices have fragmented knowledge structures, where ideas are clustered with localized links that are mostly situated in familiar contexts [4-9]. Therefore, novices usually adopt problemsolving strategies that rely on memorized processes cued by surface features, and immediately search for an equation by matching the given information with possible related variables $[4,5]$.

As students transition from novices to experts, they gain and organize new ideas, making connections among them to form a more integrated knowledge structure, a process commonly studied in the perspective of knowledge integration [11-15]. In accordance with the knowledge integration theory, when students establish an interconnected knowledge structure, a central idea becomes pivotal as the anchor point that connects all the other ideas, and thus a central node to a hierarchically organized knowledge structure [11-14]. Experts can prompt a set of central ideas through a wide range of contexts when solving problems [9-12]. The application of the central idea is not only a key feature that distinguish experts and novices commonly used in assessments [16-18], but also a crucial factor of emphasis that can aid student's to develop deep conceptual understanding [17].

One of the pioneer studies on differences in problemsolving behaviors between experts and novices was conducted by Chi et al. [5] in 1981, in which 8 experts (graduate students in physics) and 8 novices (undergraduate students in introductory physics courses) were asked to categorize physics problems based on the similarity of solutions by sorting index cards. It was found that in the 
sorting process, expert students classified problems based on the underlying physical principles that are central to solving the problem, while the novice students were often influenced by a wide range of surface features of the problems. These results suggest that students' problemsolving behaviors are influenced by their knowledge structures. For expert students who have integrated knowledge structures organized around a few central ideas, they would apply these central ideas in problem solving, which is invariant over different contextual settings of the problems. Meanwhile, novice students have fragmented knowledge structures with only local connections that are mostly situated in familiar contexts, and therefore, these students typically rely on memorized procedures cued by specific features of the contexts. The difference between novices' and experts' categorization of physics problems is also supported by cluster analysis conducted by Veldhuis [19]. In actual measurement, the choice of problems is also a very important factor. For example, Wolf, Dougherty, and Kortemeyer used a large set of problems to probe the most important properties in problem sets that discriminate experts from novices. They found that "not only was what you ask important, but also how you ask it" [20].

Utilizing this key difference in problem-solving strategies between experts and novices, instead of asking the students to solve the entire problem, Mason and Singh [21] used open-ended categorization tasks to distinguish experts from novices on a larger scale, and showed that the results from these tasks can be a reliable indicator of expertise in problem solving. In their study, the students are provided with a paper-and-pencil task to categorize kinematics and mechanics problems based on students' perceived similarities among the problems. The students were asked to write down the category names and a description of the categories, while listing the problems placed in the categories. The study has shown a wide distribution of expertise among the introductory and graduate students [21]. Following Mason and Singh's study, Zhu and Wang [22] adapted the problem sets to perform a pseudolongitudinal study on Chinese high school students' categorization of kinematics and mechanics problems, demonstrating that most students performed at the novice level, with a gradual increase in expertise as they received more training in problem solving. Other studies have also used categorization tasks to probe students' expertise in topics including electricity and magnetism [23] and quantum mechanics [24].

Although categorization tasks have been shown to be a useful assessment method in physics education research, a few improvements are also called for. In existing research, categorization tasks are mostly delivered in an open-ended format $[21,22]$, which often requires great effort on the researchers' end for coding and validating student answers, making it difficult to be widely used in large-scale studies [25]. In contrast, multiple-choice questions are graded automatically with a definitive rubric for scoring [26], and thus are commonly adopted in large-scale assessment of student learning [26,27]. However, by restricting the students to a limited number of choices, multiple-choice questions may not fully capture the spectrum of student thinking and can have nontrivial uncertainties from guessing [28]. In addition, studies on equity issues have also identified gender gaps in multiple-choice assessments of physics $[29,30]$. Nevertheless, due to its many benefits, the method of multiple-choice assessment is widely used in education research and teaching.

Hence, a number of studies on evaluation of students' problem-solving skills have been devoted to investigating whether parallel multiple-choice and free-response assessment can yield equivalent results, and most of their conclusions have been positive [26,31,32]. For instance, Hudson et al. [31] have found a strong correlation between 4 multiple-choice exams and a "post-test" with 14 freeresponse questions, especially for the case where the number of multiple-choice questions are greater. Scott et al. [32] have also found that the results from the multiplechoice questions are highly consistent with the graded explanation in a large introductory electricity and magnetism course. Based on these results, Lin and Singh [26] found that when different choices in the multiple-choice questions are weighed differently, the score of a multiplechoice test can approximate the result of a free-response test with excellent agreement. Given the promising results in many physics assessments alike, it would be useful to investigate if parallel multiple-choice and free-response categorization tasks can provide equivalent assessment. In this study, the traditional open-ended categorization tasks are adapted to a multiple-choice format, and the effectiveness of this new assessment method is evaluated.

Another assessment design feature that is investigated in this study is the influence of including diagrams in problems on student performance. In the literature, there are mixed research outcomes in this area. According to a few studies on active processing of multimedia instruction $[33,34]$ and Mayer's multimedia effect [35], students can understand the concepts more deeply from words paired with pictures than from words alone. It has been inferred that when parallel verbal and visual channels are utilized to convey information, considerably fewer cognitive resources are required to reconstruct the meaning of the context. In addition, multiple channels of information also provide a mechanism of consistency check among the different channels to improve the accuracy of the constructed meaning. All of these can lead to more accurate cognitive processing and freeing up more cognitive capacity that can be delegated to problem solving.

However, in a massive open online course experiment, Chen et al. [36] found that for many physics problems, the inclusion of a diagram has little benefit in improving students' problem-solving process, and reduces the tendency for students to draw their own. Chen et al. [36] 
argued that the advantages of Mayer's studies are related to instruction and learning, instead of problem solving. Similarly, Maries et al. [37] found that some students who did not draw a diagram and were not provided with any diagrams did better than the students who were given diagrams. In light of these differences, this study examines the influence of diagrams on students' performance in categorization tasks.

Building off the literature, two main research questions are investigated in this study:

- Can a multiple-choice format of categorization test perform equivalently to its parallel free-response categorization test?

- What is the extent to which providing diagrams in categorization tasks can influence students' performances?

\section{METHODS AND DESIGN}

\section{A. Design of the categorization tests}

In responding to the research questions, three versions of the categorization assessment have been developed, which are referred to as test $A, B$, and $C$. Test $A$ is open ended, while tests $B$ and $C$ are multiple choice. All three versions contain the same 15 questions on kinematics and mechanics, adapted from Zhu et al. [22]. Among these 15 questions, five are from Chi et al. [1] on topics of "rotational kinematics," "mechanical energy conservation," "Newton's second law," and "work-energy theorem"; three are from Mason et al. [21] on topics of "2D kinematics" and "conservation of momentum + conservation of energy." The remaining 7 questions are adapted from past Chinese college entrance exams on topics of rotational kinematics, 2D projectile motion, and conservation of momentum + conservation of energy.

To design the multiple-choice versions, the student reported categories in Zhu et al. [22] are analyzed and ranked based on their popularities, which give a list of a

(a)7. A ball is thrown from the top of a $35 \mathrm{~m}$ high building with an initial speed of $80 \mathrm{~m} / \mathrm{s}$ at an angle of $25^{\circ}$ above the horizontal. Find the time it takes to reach the ground. total of 29 popular categories. In addition, two more categories on "momentum theorem" and rotational kinematics are added, which did not appear in Zhu et al.[22] but are considered as necessary for completing the possible set of categories by researchers of this study. Then the entire list of 31 categories are used as choices in the multiple-choice versions. Test $B$ is designed as a parallel test of test $A$. Both versions use the same question narratives and presentations except that test $A$ is open ended and test $B$ is multiple choice.

Among the 15 questions in tests $A$ and $B, 10$ questions do not have diagrams. A third test version (test $C$ ) is then generated based on test $B$ by adding diagrams to each of the 10 questions that do not have diagrams originally, while keeping the other 5 questions the same. For instance, question 7 does not have a diagram in test $B$, but it has one in test $C$ as shown in Fig. 1. All the questions between tests $B$ and $C$ have identical tasks and narratives. By comparing students' responses on the 10 questions that differ in diagrams between tests $B$ and $C$, the influence of diagrams on students' performances on multiple-choice categorization tasks can be studied.

For all three versions, the instructions for the categorization tasks are clearly laid out on the front page, informing the students to analyze the problems without solving them and to categorize them based on the basic features of the problems. The open-ended assessment (test $A$ ) requires the students to write down the names of their categories, the questions in each category, and a brief explanation of their reasoning, which is a procedure identical to that in Zhu et al. [22]. On the multiple-choice assessments (tests $B$ and $C$ ), students are asked to choose and mark up to three relevant categories from a given list. Examples of the answer sheets are shown in Fig. 2. Note that the options in the answer sheet are generally organized by text-length to make the layout fit. It appears that the terms for surface features are shorter in length compared to the descriptions of the key principles.

(b)7. A ball is thrown from the top of a $35 \mathrm{~m}$ high building with an initial speed of $80 \mathrm{~m} / \mathrm{s}$ at an angle of $25^{\circ}$ above the horizontal. Find the time it takes to reach the ground.

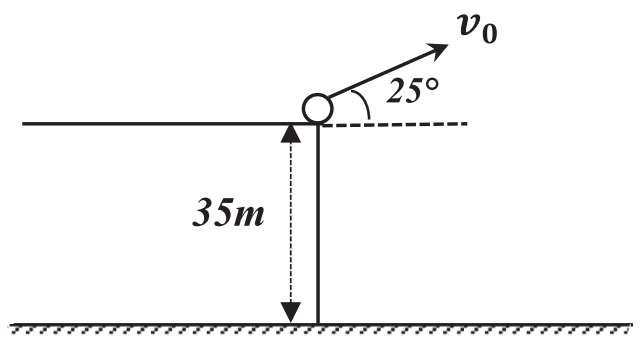

FIG. 1. Question 7 in tests $B$ and $C$ : (a) without a diagram in test $B$, and (b) with a diagram in test $C$. 
Answer Sheet of Test A

(a)

Category Name:

Question ID:

Reason:

Answer Sheet of Test B/C

(b)

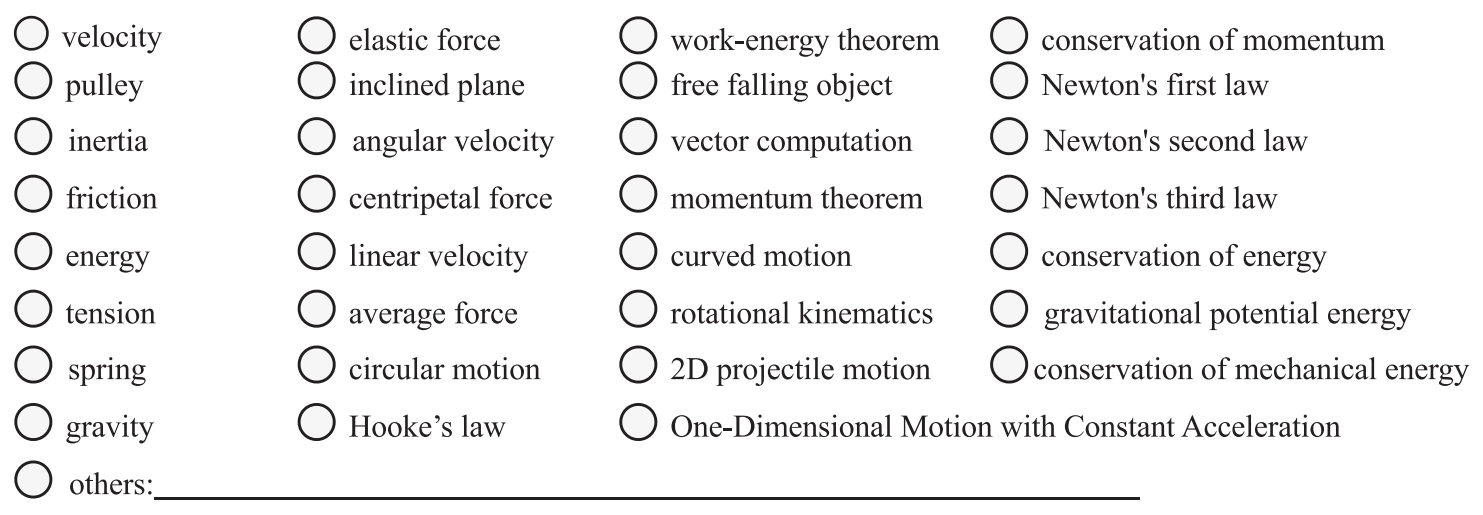

FIG. 2. The answer sheet section: (a) In test $A$, students are asked to write down up to 7 categories. The section below shows the answer section for one category, which is repeated 7 times on the answer sheet; (b) tests $B$ and $C$.

\section{B. Research procedure}

The subjects of this study were Chinese college students at a high-ranking public university (top 35 in China). The students came from a variety of majors, including chemistry, medical science, and biology. They were taking the calculus-based sequence of introductory physics course on topics of mechanics, electricity and magnetism, and waves. All students were asked to complete the assessment on their own within $45 \mathrm{~min}$. The assessment was conducted after the students had completed their learning on the mechanics part of the course. All students took the Chinese versions of the tests. An English translation of test $C$ is included in the Supplemental Material [38].

Two studies have been conducted to answer the two research questions outlined in the previous section. Study 1 investigates the consistency between the open-ended and multiple-choice categorization tasks. Test $A$ and test $B$ were administered in two classes of the physics course in the same week. The students in the two classes were from different departments so there was little chance that the students would communicate about their tests. In each class, tests $A$ and $B$ were evenly mixed so that the students in adjacent seats received randomly different versions of the test. Students had 45 min to answer the test and most of the students finished the test around half an hour. Altogether 45 valid responses for test $A$ and 37 for test $B$ were collected. The results from the two tests are compared to evaluate the extent to which the two assessment methods can produce similar outcomes.

Study 2 examines the influence of diagrams on students' performance on categorization tasks. In study 2 , test $B$ and test $C$ were also mixed and randomly distributed to students in a third class of calculus-based physics different from the two classes involved in study 1. A total of 49 valid responses for test $B$ and 48 for test $C$ were collected in study 2 . The results from the 10 questions with and without diagrams in the two tests are compared to analyze the influence of the diagrams on students' performances in the multiple-choice form of categorization tasks. The results of the remaining 5 identical questions (all with diagrams) are used to evaluate the equivalence of the two student groups. In order to maintain the comparability of the student groups in content learning, all testing involved in study 1 and study 2 was administered in the same week. 


\section{Grading rubric}

\section{Open-ended assessment (test A)}

For evaluating the open-ended categorization test (test A), similar grading rubrics were adopted from Mason et al. [21] and Zhu et al. [22]. Students' responses for each question were evaluated as good, moderate, or poor regarding problem categorization. Table I gives a list of categorization responses considered to be good, moderate, and poor for each of the 15 questions. The scoring of test $A$ was conducted by two researchers. Any scoring discrepancies were discussed and resolved in group meetings.

In the grading rubric, good categorization responses are the ones corresponding to the relevant physics principles considered by experts as essential to correctly solve the problem. When a problem is related to two or more different concepts, a good category needs to include a combination of the relevant physical concepts or principles. For example, as can be seen in Table I, questions 2 and 4 involve multiple concepts, and one of the good categories, namely, Newton's second law and kinematics, is a combination of two different physics concepts.

Moderate category responses are the ones that are too broad to accurately classify the question (such as velocity as opposed to 2D kinematics for question 10), and those that include only part of the related concepts. For example, energy conservation and momentum conservation is a good category for question 13 . If a student only classifies this item with the momentum conservation, then this response will be considered as moderate.

Poor category responses are the ones that correspond to surface features of the problem (such as "pulley" and "spring" for questions 2, 6, and 9) and irrelevant physics concepts. Therefore, a concept can be good for one question, but poor for another. For example, mechanical energy conservation is a good category for question 8 , but a poor one for question 13 .

Once a response on a question is classified as being good, moderate, or poor, numerical points are assigned based on its categorization qualities: 2 points for good, 1 point for moderate, and 0 for poor. For test $A$, which has 15 questions, the range of the total score a student can obtain is from 0 to 30 points.

\section{Multiple-choice versions (tests B and C)}

For evaluating the multiple-choice tests, all the category choices were carefully evaluated by eight physics experts including one faculty, two Ph.D. candidates, and five master students majoring in physics education research.

TABLE I. Evaluation rubric for the open-ended categorization test with classifications considered as good, moderate, and poor for each question. N/A means that no related classifications were identified as matching the performance category for that question.

\begin{tabular}{|c|c|c|c|}
\hline & Good & Moderate & Poor \\
\hline 1 & Rotational kinematics & Rigid body & Centripetal force \\
\hline 2 & $\begin{array}{l}\text { Mechanical energy conservation, work-energy } \\
\text { theorem, definition of work, Newton's second } \\
\text { law and kinematics }\end{array}$ & Newton's second law & Pulley, freely falling body \\
\hline 3 & Newton's second law & Force analysis & Force \\
\hline 4 & Newton's second law and circular motion & Circular motion & Angular velocity \\
\hline 5 & $\begin{array}{l}\text { Mechanical energy conservation, 2D kinematics } \\
\text { and vector computation, work-energy theorem }\end{array}$ & $\mathrm{N} / \mathrm{A}$ & Velocity \\
\hline 6 & $\begin{array}{l}\text { Work-energy theorem, Newton's second law and } \\
\text { kinematics }\end{array}$ & Newton's second law & Spring, distance \\
\hline 7 & $\begin{array}{l}\text { 2D kinematics, vector computation, Newton's } \\
\text { second law and kinematics }\end{array}$ & $\mathrm{N} / \mathrm{A}$ & Average velocity \\
\hline 8 & $\begin{array}{l}\text { Mechanical energy conservation and momentum } \\
\text { conservation, work-energy theorem, and } \\
\text { momentum conservation }\end{array}$ & Mechanical energy conservation & Height \\
\hline 9 & $\begin{array}{l}\text { Mechanical energy conservation and Impulse- } \\
\text { Momentum theorem, work-energy theorem, and } \\
\text { Impulse-momentum theorem }\end{array}$ & Mechanical energy conservation & Pulley, changed force \\
\hline 10 & 2D kinematics & Velocity & Motion stage \\
\hline 11 & Newton's second law and circular motion & Friction & Vector computation \\
\hline 12 & 2D kinematics, vector computation & $\mathrm{N} / \mathrm{A}$ & Gravitational potential energy \\
\hline 13 & Energy conservation and momentum conservation & Energy & Mechanical energy conservation \\
\hline 14 & $\begin{array}{l}\text { Linear motion with constant acceleration and } \\
\text { Newton's second law, work-energy theorem }\end{array}$ & Newton's second law & Ramp, circular motion \\
\hline 15 & $\begin{array}{l}\text { Mechanical energy conservation, work-energy } \\
\text { theorem, uniformly accelerated linear motion } \\
\text { and Newton's second law }\end{array}$ & Linear motion with constant acceleration & Resolution of force \\
\hline
\end{tabular}


TABLE II. Answer choices for the multiple-choice categorization tests classified by experts into the concept group and the context group.

Concept Centripetal force, work-energy theorem, circular motion, free falling object, Hooker's law, rotational kinematics, impulsemomentum theorem, momentum conservation, Newton's first law, Newton's second law, Newton's third law, 2D kinematics, energy conservation, linear motion with constant acceleration, conservation of mechanical energy, inertia, vector computation, curved motion

Context Speed, elastic force, gravity, ramp, tension, spring, pulley, energy, angular velocity, linear velocity, average force, friction, gravitational potential energy

For the 31 choices in tests $B$ and $C$, the eight experts voted on each option about its categorization basis, i.e., whether the option reflected a surface feature or a physical principle. In the voting, there were divergent opinions about several options such as "circular motion," "curvilinear motion," and "energy." Agreements were finally reached after analyzing students' answers to the related questions. The classification of the options used in tests $B$ and $C$ is listed in Table II.

For each question, students can choose up to three choices from the list shown in Table II. This option of giving multiple answers was designed to better probe the possible mixed states of student understanding that may hold multiple views of the concept. However, the freedom of giving multiple answers also leads to new aspects of measurement. For the open-ended test, students were sorting the questions into different categories, and in doing so, they typically assigned a question in only one category defined by themselves. As a result, it was very uncommon for a student to categorize a question into both correct and incorrect categories. Therefore, a positive 0 to 2 scale worked quite well for scoring the open-ended responses. On the other hand, for the multiple-choice test, since the choices are a limited subset of popular definitions, they

TABLE III. Examples of popular wrong concepts for each question in the multiple-choice assessment. N/A represents that there are no wrong concepts for this question among the choices.

\begin{tabular}{ll}
\hline \hline & \multicolumn{1}{c}{ Wrong concept } \\
\hline 1 & Circular motion \\
2 & Rotational kinematics \\
3 & Conservation of mechanical energy \\
4 & Rotational kinematics \\
5 & $\mathrm{~N} / \mathrm{A}$ \\
6 & Conservation of mechanical energy \\
7 & Free falling object \\
8 & $\mathrm{~N} / \mathrm{A}$ \\
9 & Momentum conservation \\
10 & $\mathrm{~N} / \mathrm{A}$ \\
11 & Momentum conservation \\
12 & Momentum conservation \\
13 & Mechanical energy conservation \\
14 & Mechanical energy conservation \\
15 & Rotational kinematics \\
\hline \hline
\end{tabular}

may not best fit individual students' own thinking. Therefore, it was common for a student to choose multiple answers for a question, which might include both correct and incorrect choices. In such cases, using both positive and negative points becomes a useful coding approach to account for the mixed thoughts of students who were in the transitional stage between novices and experts.

In responding to the new features of the multiple-choice test, the grading rubric was modified compared to the one for the open-ended questions. Specifically, a student was given 2 points for a good category, 1 point for a moderate category, and 0 for a poor category, which is similar to the open-ended rubric. However, if a student chose an incorrect concept or a context choice, negative points were assigned. For each question, 1 point was subtracted for each wrong concept, and 0.5 points were subtracted for each context choice. The list of wrong concepts for each question is shown in Table III. For example, as can be seen from Table III, the good category for question 13 is a combination of energy conservation and momentum conservation. Therefore, according to the rubric, if a student chooses the categories momentum conservation (one of the relevant concept), mechanical energy conservation (wrong concept), energy (context), and velocity (context), then this student will receive $1-1-2 \times 0.5=-1$ points for question 13. For each question the maximum negative points will be set at -2 . Therefore, for the 15-question test, the range of the total score is -30 to 30 points.

The differences in test forms and grading rubrics will lead to different scores produced. Since the main purpose of this study is to develop a multiple-choice version of the categorization test, the grading rubric is designed to best address the unique features of the student responses of the multiple-choice test. Although the rubrics are different, the scores from the two tests can be converted into the same scale for comparisons.

\section{ANALYSIS AND RESULTS}

\section{A. Results of study 1}

To evaluate the equivalence between the open-ended and multiple-choice versions of categorization tests, students' scores on tests $A$ and $B$ are compared. According to the scoring rubric detailed in the previous section, the range of scores of a question in test $A$ is 0 to 2, while a question in 
(a)

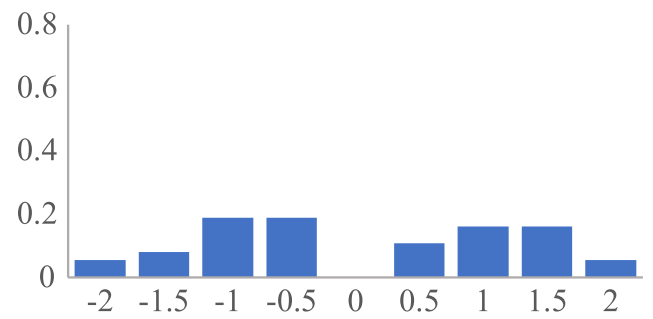

(b)

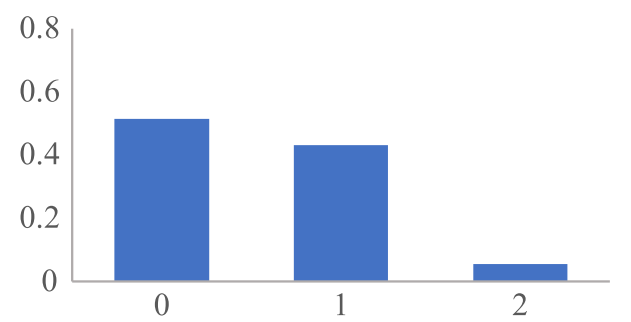

FIG. 3. Score distribution for question 1 of test $B$ (a) before the conversion, and (b) after the conversion. The horizontal axis represents the range of scores for a question, and the vertical axis represents the fraction of students receiving that score.

(a)

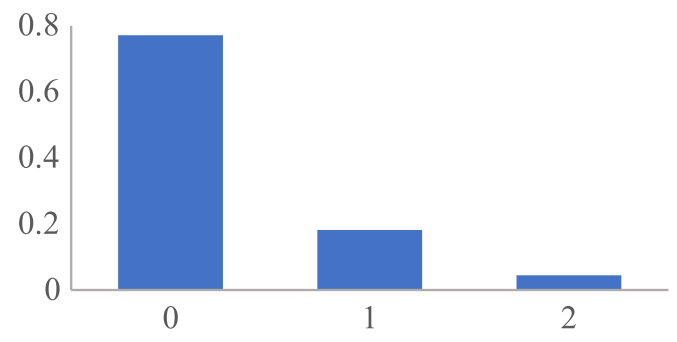

(b)

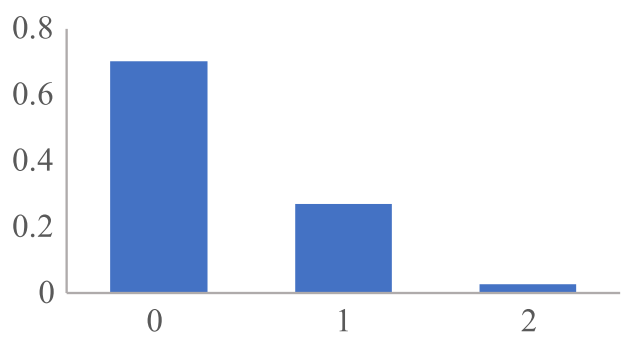

FIG. 4. Score distribution of question 14 in (a) version $A$, and (b) version $B$. The horizontal axis represents the range of scores for a question, and the vertical axis represents the fraction of students receiving that score.

test $B$ is scored from -2 to 2 . Therefore, in order to compare students' scores on the two tests, it is necessary to convert these two scores into a single scale. In this analysis, the score of a test $B$ question is converted into the scale of a test $A$ question, which ranges from 0 to 2 . For score conversion of test $B$ questions, a score less than or equal to 0.5 is converted to 0 , a score larger than 0.5 and less than 1.5 is converted to 1 , and a score greater than 1.5 is converted to 2 points. As an illustration, the score distribution for question 1 before and after the conversion is shown in Fig. 3.

After converting to the same scale, corresponding questions on the two tests are paired for comparison. As an illustration, the score distributions for question 14 in tests $A$ and $B$ are shown in Fig. 4, which show similar distribution patterns. To quantitatively evaluate the similarity between the scores of the two versions of test, the average scores of the two tests are calculated and plotted in Fig. 5. The results show that the two tests produce nearly identical scores ( $p=0.883, d f=78, d=0.032$ ).

To evaluate the overall consistency of measurement of the questions in the two tests, a scatter plot of the average scores of each question between the two tests is graphed along with a linear regression line in Fig. 6. The result shows a large correlation coefficient of $0.839(p=0.000)$, indicating high consistency between questions in tests $A$ and $B$. For the correlation analysis, the score distributions of the two tests were evaluated for the normality test using a $Q-Q$ plot and confirmed to follow the normal distribution.

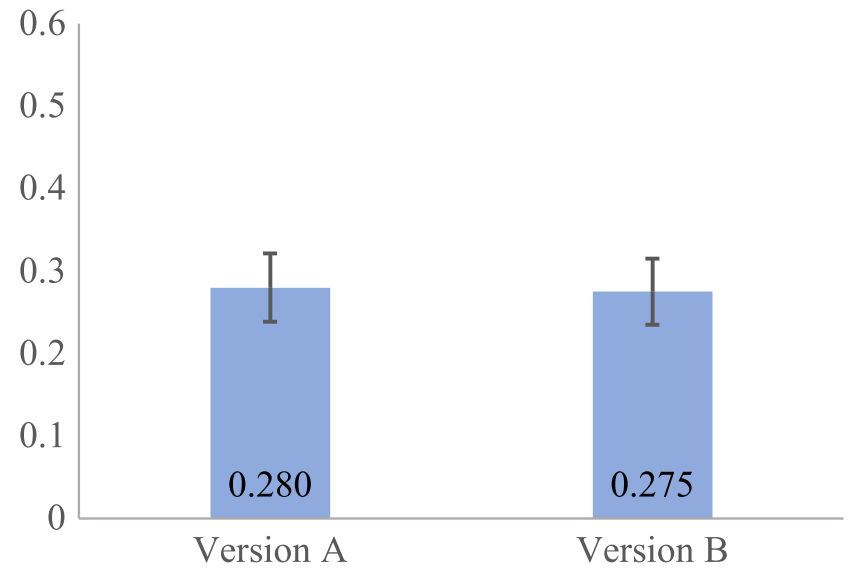

FIG. 5. Mean scores of tests $A$ and $B$. The error bars represent standard errors (SE). The scores are scaled from 0 to 1 .

To further evaluate if the two versions of categorization tests would respond consistently with students at different levels of class performance, students are grouped into high (top 50\%) and low (bottom 50\%) performance groups based on their scores on their physics midterm exam. For the students who took test $A$, the high and low performance group's score on the midterm was $85.9 \%$ and $68.0 \%$, respectively. Similarly, for the students who took test $B$, the high and low performance group's score on the midterm was $86.8 \%$ and $69.7 \%$, respectively. The results show that the high and low groups of the students taking the two 


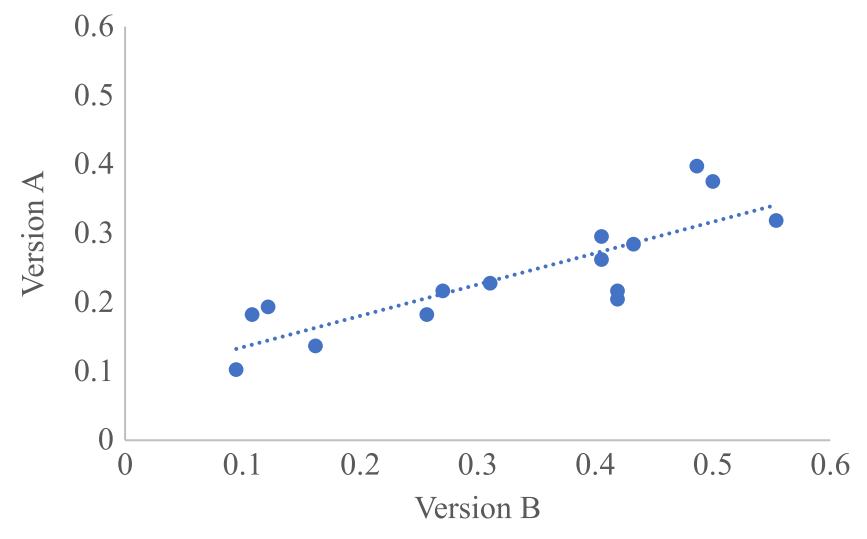

FIG. 6. Correlation of the average scores of each question between tests $A$ and $B$. The horizontal axis is the average score of a question in test $B$, and the vertical axis is the average score of the corresponding question in test $A$. The scores are scaled from 0 to 1 . The error of the linear fit is $\mathrm{RMSE}=0.043$, which is at a similar level of the standard error of the average score of a question $(\mathrm{SE} \approx 0.05)$.

versions of the test are statistically indistinguishable $\left(t_{\text {high }}=0.866\right.$ and $\left.t_{\text {low }}=0.307\right)$, which suggests good consistency between the two randomly assigned samples.

The average scores of tests $A$ and $B$ from the high and low performance groups are calculated and plotted in Fig. 7. The results show that the high and low groups of students have very similar performance on the two versions of the test. The performance differences between the two groups' test scores are also very similar. A two-way ANOVA analysis suggests that there was a main effect due to the high-low grouping by exam scores $(p=0.032)$, while the effect due to test versions $(A$ and $B)$ is insignificant $(p=0.361)$. In addition, there was no interaction effect between high-low groups and test versions $(p=0.705)$. The results further confirm that the two categorization tests provide consistent assessment among high and low performing students. Therefore, the multiplechoice version can be used as an alternative assessment of

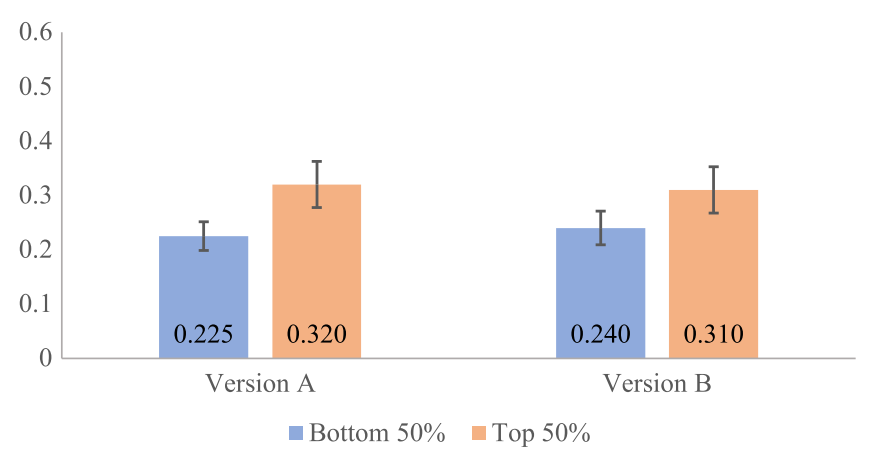

FIG. 7. Average scores on tests $A$ and $B$ of the top and bottom $50 \%$ of the students according to their midterm exam scores. The error bars represent standard errors. The scores are scaled from 0 to 1 . problem categorization, which provides equivalent results with improved convenience compared to the open-ended version.

\section{B. Results of study 2}

In order to examine the influence of diagrams on student performance in categorization tasks, the average score of the 10 questions without diagrams in test $B$ is compared with the average score of the corresponding 10 questions with diagrams in test $C$. The remaining 5 identical questions in both versions are used to establish the equivalence of the two groups of students taking test $B$ or $C$. Figure 8 shows the mean scores of the 10 questions with varying diagram settings in tests $B$ and $C$. The results suggest that the inclusion of diagrams significantly improves the students' performances in categorization tasks $(p=0.012, d f=89, d=0.55)$.

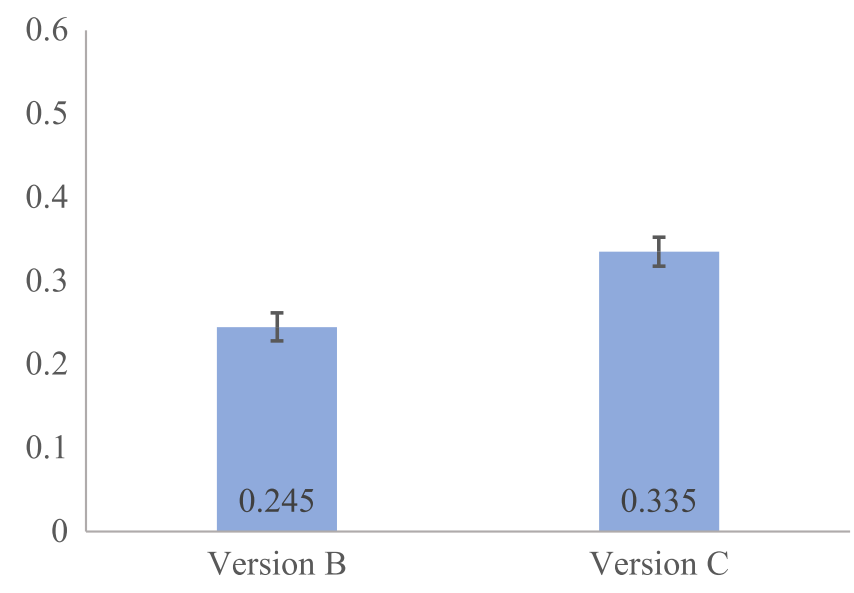

FIG. 8. Mean scores of the 10 questions in test $B$ (without diagrams) and in test $C$ (with diagrams). The error bars represent standard errors. The scores are scaled from 0 to 1 .

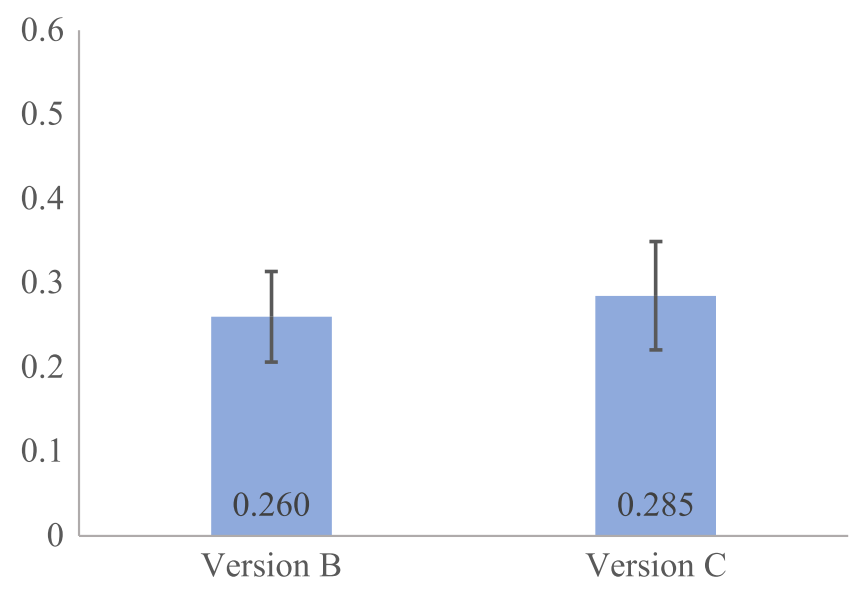

FIG. 9. Mean scores of the 5 identical questions in tests $B$ and $C$. The error bars represent standard errors. The scores are scaled from 0 to 1 . 
To compare if students taking test $B$ or $C$ are equivalent samples, students' scores on the 5 identical questions in both tests are compared in Fig. 9, which shows that the mean scores of these 5 questions are not statistically different $(p=0.51, d f=89)$. Overall, the results from this study suggest that the students taking test $B$ or $C$ are identical samples and that including diagrams can improve students' performances on problem-categorization tasks.

\section{CONCLUSIONS AND DISCUSSIONS}

In this study, the traditional open-ended categorization tasks are adapted to a multiple-choice format, and the effectiveness of this new assessment method is studied. Through comparisons of students' performances on the open-ended and multiple-choice forms of categorization tasks, it is suggested that the multiple-choice format of the categorization tasks performs just as well as the open-ended format. Therefore, the multiple-choice categorization task assessment can provide an effective alternative method, which will significantly improve the grading efficiency and help researchers save time and resources when implementing categorization tasks.

Although the multiple-choice method appears to be a useful alternative, the open-ended assessment still has its merits. Since the multiple-choice assessment limits students' choices of categorization by providing them with a predefined set of choices, if the research goal is to obtain detailed information on particular aspects of students' answers, such as it is in Zhu et al. [22], the open-ended assessment would be more appropriate. On the other hand, if the goal is to assess students' knowledge structure efficiently in large scale, the multiple-choice assessment is usually the preferred tool.

The grading rubric and score conversion used in our study generate consistent scores between the open-ended questionnaire and the multiple-choice test. However, there could be alternate scoring methods that may produce better agreement between the two types of assessments. For example, in certain situations, one may choose to use a single-response format for the multiple-choice test, which may create more consistency in overall scoring but at the expense of losing the information of possible mixed understanding. Further studies can be carried out to identify the most appropriate rubric for specific research goals by varying the related categorization parameters, response constraints, and scoring rules.

In addition to validating the multiple-choice format of the categorization test, the results from study 1 also provide an empirical assessment of student knowledge structure on introductory mechanics among the first-year college students in a high-ranking university in China. The mean scores on both tests $A$ and $B$ are at the $25 \%$ level, which suggests that many of these students are at an early transitional stage just beyond the novice level of conceptual understanding with approximately $25 \%$ chance of identifying the correct underlying principles in physics problems. As demonstrated by previous research, the primary problem-solving strategies of novice students often rely on memorized fragments based on surface features of the problems. Aided with the quantitative assessment approach of problem categorization and combined with other research methods such as interviews and conceptual surveys, further research can be conducted to investigate fine-grained states of student knowledge structures and developmental pathways during learning, which can provide useful insight in developing effective instructional strategies that promote knowledge integration and deep understanding.

This study also examines the influence of diagrams on student performance in categorization tasks. It has been shown that the inclusion of diagrams in problems can improve the chance of students choosing the relevant concepts. This result agrees with Mayer's multimedia effect [35], which also advocates the use of diagrams, confirming that diagrams can improve student performance in the early stages of problem solving.

\section{ACKNOWLEDGMENTS}

The research is supported in part by the National Science Foundation Grant No. DUE-1712238, by the Joint Research Institute for Science and Society (JoRISS), and by the East China Normal University (2020ECNUXFZH001). Any opinions, findings, and conclusions or recommendations expressed in this paper are those of the authors and do not necessarily reflect the views of the funding agencies.
[1] M. Alonso, Problem solving vs conceptual understanding, Am. J. Phys. 60, 777 (1992).

[2] L. Bao and E. F. Redish, Model Analysis: Assessing the dynamics of student learning, Phys. Rev. ST Phys. Educ. Res. 2, 010103 (2006).
[3] L. Bao and K. Koenig, Physics education research for 21st century learning, Discip. Interdiscip. Sci. Educ. Res. 1, 1 (2019).

[4] A. B. Champagne, R. F. Gunstone, and L. E. Klopfer, A perspective on the differences between expert and novice 
performance in solving physics problems, Res. Sci. Educ. 12, 71 (1982).

[5] M. T. Chi, P. J. Feltovich, and R. Glaser, Categorization and representation of physics problems by experts and novices, Cogn. Sci. 5, 121 (1981).

[6] W. J. Gerace, R. J. Dufresne, W. J. Leonard, and J. P. Mestre, Problem solving and conceptual understanding, in Proceedings of the 2001 Physics Education Research Conference, Rochester, NY, edited by K. Cummings, S. Franklin, and J. Marx (AIP, New York, 2001), https:// doi.org/10.1119/perc.2001.inv.005.

[7] B. S. Eylon and F. Reif, Effects of knowledge organization on task performance, Cognit. Instr. 1, 5 (1984).

[8] A. H. Schoenfeld and D. J. Herrmann, Problem perception and knowledge structure in expert and novice mathematical problem solvers, J. Exper. Psychol. 8, 484 (1982).

[9] J. L. Snyder, An investigation of the knowledge structures of experts, intermediates and novices in physics, Int. J. Sci. Educ. 22, 979 (2000).

[10] J. Larkin, J. McDermott, D. P. Simon, and H. A. Simon, Expert and novice performance in solving physics problems, Science 208, 1335 (1980).

[11] H. S. Lee, O. L. Liu, and M. C. Linn, Validating measurement of knowledge integration in science using multiple-choice and explanation items, Appl. Meas. Educ. 24, 115 (2011).

[12] M. C. Linn, The Knowledge Integration Perspective on Learning and Instruction, The Cambridge Handbook of: The Learning Sciences (Cambridge University Press, New York, 2005), pp. 243-264, https://doi.org/10.1017/ cbo9780511816833.016.

[13] J. Shen, O. L. Liu, and H. Y. Chang, Assessing students' deep conceptual understanding in physical sciences: an example on sinking and floating, Int. J. Sci. Math. Educ. 15, 57 (2017).

[14] M. Kubsch, J. Nordine, K. Neumann, D. Fortus, and J. Krajcik, Measuring integrated knowledge-a network analytical approach, in Proceedings of the 13th International Conference of the Learning Sciences (ICLS), London, United Kingdom (International Society of the Learning Sciences, Inc. [ISLS], London, United Kingdom, 2018), https://repository.isls.org//handle/1/627.

[15] J. Nordine, J. Krajcik, and D. Fortus, Transforming energy instruction in middle school to support integrated understanding and future learning, Sci. Educ. 95, 670 (2011).

[16] R. Dai, J. C. Fritchman, Q. Liu, Y. Xiao, and L. Bao, Assessment of student understanding on light interference, Phys. Rev. Phys. Educ. Res. 15, 020134 (2019).

[17] Y. Nie, Y. Xiao, J. C. Fritchman, Q. Liu, J. Han, J. Xiong, and L. Bao, Teaching towards knowledge integration in learning force and motion, Int. J. Sci. Educ. 41, 2271 (2019).

[18] W. Xu, Q. Liu, K. Koenig, J. Fritchman, J. Han, S. Pan, and L. Bao, Assessment of knowledge integtration in student learning of momentum, Phys. Rev. Phys. Educ. Res. 16, 010130 (2020).

[19] G. H. Veldhuis, The use of cluster analysis in categorization of physics problems, Sci. Educ. 74, 105 (1990).

[20] S. F. Wolf, D. P. Dougherty, and G. Kortemeyer, Rigging the deck: Selecting good problems for expert-novice cardsorting experiments, Phys. Rev. ST Phys. Educ. Res. 8, 020116 (2012).
[21] A. Mason and C. Singh, Assessing expertise in introductory physics using categorization task, Phys. Rev. ST Phys. Educ. Res. 7, 020110 (2011).

[22] G. Zhu and J. Wang, Pseudolongitudinal investigation on Chinese students' categorization of kinematics and mechanics problems, Phys. Rev. Phys. Educ. Res. 13, 020118 (2017).

[23] E. R. Savelsbergh, T. d. Jong, and M. G. Ferguson-Hessler, Choosing the right solution approach: The crucial role of situational knowledge in electricity and magnetism, Phys. Rev. ST Phys. Educ. Res. 7, 010103 (2011).

[24] S.-Y. Lin and C. Singh, Categorization of quantum mechanics problems by professors and students, Eur. J. Phys. 31, 57 (2009).

[25] H. Wainer and D. Thissen, Combining multiple-choice and constructed-response test scores: Toward a Marxist theory of test construction, Appl. Meas. Educ. 6, 103 (1993).

[26] S.-Y. Lin and C. Singh, Can free-response questions be approximated by multiple-choice equivalents?, Am. J. Phys. 81, 624 (2013).

[27] L. Ding and R. Beichner, Approaches to data analysis of multiple-choice questions, Phys. Rev. ST Phys. Educ. Res. 5, 020103 (2009).

[28] R. M. Harden, R. A. Brown, L. A. Biran, W. D. Ross, and R. E. Wakeford, Multiple choice questions: To guess or not to guess, Med. Educ. 10, 27 (1976).

[29] K. Wilson, D. Low, M. Verdon, and A. Verdon, Differences in gender performance on competitive physics selection tests, Phys. Rev. Phys. Educ. Res. 12, 020111 (2016).

[30] R. Henderson, G. Stewart, J. Stewart, L. Michaluk, and A. Traxler, Exploring the gender gap in the conceptual survey of electricity and magnetism, Phys. Rev. Phys. Educ. Res. 13, 020114 (2017).

[31] H. T. Hudson and C.K. Hudson, Suggestions on the construction of multiple-choice tests, Am. J. Phys. 49, 838 (1981).

[32] M. Scott, T. Stelzer, and G. Gladding, Evaluating multiplechoice exams in large introductory physics courses, Phys. Rev. ST Phys. Educ. Res. 2, 020102 (2006).

[33] M. C. Wittrock, Generative processes of comprehension, Educ. Psychol. 24, 345 (1989).

[34] R. Moreno and R. E. Mayer, Cognitive principles of multimedia learning: The role of modality and contiguity, J. Educ. Psychol. 91, 358 (1999).

[35] R. E. Mayer, Cognitive theory of multimedia learning, in The Cambridge Handbook of Multimedia Learning (Cambridge University Press, Cambridge, United Kingdom, 2005), p. 3148, https://doi.org/10.1017/cbo978 1139547369.005.

[36] Z. Chen, N. Demirci, Y.-J. Choi, and D. E. Pritchard, To draw or not to draw? Examining the necessity of problem diagrams using massive open online course experiments, Phys. Rev. Phys. Educ. Res. 13, 010110 (2017).

[37] A. Maries and C. Singh, Should students be provided diagrams or asked to draw them while solving introductory physics problems?, AIP Conf. Proc. 1413, 263 (2012).

[38] See Supplemental Material at http://link.aps.org/ supplemental/10.1103/PhysRevPhysEducRes.16.020120 for the multiple-choice version of categorization test and its answer sheet. 